\title{
Flare Stars Database: Present Status
}

\author{
K.P. Tsvetkova, M.K. Tsvetkov, K.Y. Stavrev \\ Institute of Astronomy, Bulgarian Academy of Sciences, \\ Tsarigradsko Shose 72, BG-1784 Sofia, Bulgaria
}

\begin{abstract}
An updated data collection for UV Cet type stars and their registered flare events in stellar aggregates and in the solar neighbourhood during more than 9580 hours observing time is described. The Flare Stars Database (FSDB) presently includes 3127 entries for 1622 stars.
\end{abstract}

\section{Introduction}

Among the stellar content of young star clusters and associations, stars with flare activity constitute a significant fraction. Up to February 1993, the number of known variable stars in Orion is 1380,559 of them are UV Cet stars. This indicates that flare activity is a common characteristic in the early evolution of red dwarf stars. The distribution of flare stars in stellar aggregates and in the solar neighbourhood and the same nature of both types of stars, differing only in age and mass, led to the conclusion that UV Cet type stars in the solar neighbourhood have survived their original stellar system, which has disintegrated, so that these stars belong now to the general galactic field. Strange biases exist on these stars in the literature, obviously due to the lack of extensive flare star data collections. In their overview of low mass star formation in the Orion region, Brand \& Wouterloot (1991) include only 116 of the 559 known flare stars. In order to avoid that significant numbers of low mass stars are neglected when processes of low mass star formation are considered, and in order to facilitate statistical investigations, we have collected all flare star data known to us.

\section{Status of Flare Star Observations}

Flare activity is a random time process, thus the parameters describing this process and the evaluation of the total number of flare stars in a given aggregate (those with registered flare events and those which are not yet discovered) must be based on large samples of observational material. The status of flare star observations from the beginning of systematic monitoring up to the present is shown in Table 1. Aggregates with monitoring of more than 100 hours are listed. 
Table 1. Optical flare star monitoring observations up to December 1994

\begin{tabular}{lrrr}
\hline \multicolumn{1}{c}{ Region } & $\begin{array}{c}\text { No. of } \\
\text { flare } \\
\text { stars }\end{array}$ & $\begin{array}{c}\text { Nogistered of } \\
\text { flares }\end{array}$ & $\begin{array}{c}\text { Observing } \\
\text { time } \\
\text { (hours) }\end{array}$ \\
\hline Orion M42/M43 & 563 & 826 & 1623 \\
Pleiades & 547 & 1635 & 3250 \\
Taurus dark clouds & 102 & 122 & 870 \\
Cygnus NGC 7000 & 83 & 120 & 1168 \\
Praesepe & 59 & 146 & 680 \\
Monoceros NGC 2264 & 42 & 43 & 105 \\
Cygnus IC 1318 & 17 & 18 & 300 \\
Coma open cluster & 14 & 21 & 338 \\
Scorpius-Ophiuchus & 14 & 15 & 321 \\
Alpha Persei cluster & 6 & 6 & 128 \\
Others & 70 & 70 & 800 \\
Solar neighbourhood & 105 & $>105$ & \\
\hline Total & 1622 & $>3127$ & $>9583$ \\
\hline \hline
\end{tabular}

\section{The Flare Stars Database}

In order to provide direct access to the original flare star data, we assembled catalogues, which are compilations of parameters drawn from many sources (e.g. for Cygnus, Praesepe), and we made computer-readable versions of already existing catalogues (Pleiades region: Haro et al. 1982; Orion region: Natsvlishvili 1989).

The original catalogues were checked with the help of the special programme package FLAREBASE (Tsvetkov et al. 1993). The aim was not only the preparation of master catalogues but also a critical evaluation to ensure the homogeneity of the data, which are needed for basic statistical statements, e.g. the number distribution of flare stars with stellar magnitude in quiescence or with outburst amplitude, time and energy parameters for the calculation of the total flare-up energy. FLAREBASE also helped to correct the errors in the original catalogues, to supplement the data, and to add new information.

After analysing the existing flare star data we adopted the following table structure for the FSDB (Table 2).

Each flare event is identified uniquely by its identifier, which is the primary key for searching and linking the information. The structure of the identifier is:

\begin{tabular}{|c|c|c|c|}
\hline 5 bytes & 3 bytes & 6 bytes & 1 byte \\
\hline $\begin{array}{c}\text { Name of the star } \\
\text { according to GCVS }\end{array}$ & Constellation & $\begin{array}{c}\text { Date of the } \\
\text { flare event }\end{array}$ & $\begin{array}{c}\text { Consecutive Nos. of events } \\
\text { occurring on the same date }\end{array}$ \\
\hline
\end{tabular}

For instance, $\mathrm{CU}_{\sqcup \sqcup \sqcup} \mathrm{CNC7301302}$ identifies the second flare event on 30 January 1973 on the star CU Cnc. 
Table 2. Structure of the FSDB

MAIN TABLE

$\downarrow$

\begin{tabular}{|c|}
\hline Flare event identifier \\
\hline Equatorial coordinates (R.A., Decl.) of flare star for equinox J2000.0 \\
\hline Equatorial coordinates (R.A., Decl.) of flare star for equinox B1950.0 \\
\hline Galactic coordinates in the l(II), b(II) system \\
\hline Julian date of the flare event \\
\hline First registered flare event \\
\hline Telescope used \\
\hline Stellar magnitude at minimum in $U$ or pg/POSS $B$ bands \\
\hline Stellar magnitudes at maximum in $U$ and pg bands \\
\hline Amplitudes in $U$ and pg bands \\
\hline Criterion - Dm $>5$ s \\
\hline$V$ magnitude, $B-V$ and $U-B$ indices at minimum \\
\hline Spectral class \\
\hline Aggregate membership \\
\hline Pointers to tables SIMBAD and NOTES \\
\hline
\end{tabular}

$\downarrow \downarrow \downarrow \downarrow$

\begin{tabular}{|c|}
\hline Table CROSS-IDENTIFICATION \\
\hline Table REFERENCES \\
\hline Table SIMBAD \\
\hline Table NOTES \\
\hline
\end{tabular}

All preparatory work on the FSDB has been executed on the IBM/4381 main frame computer at the Computer Centre of Physics of the Bulgarian Academy of Sciences, using the facilities of the operational system VM/SP Rel. 4. The FSDB is a database not only for UV Cet type stars but also for their registered flare events, which are missing in other astronomical catalogues and data sets: the General Catalogue of Variable Stars (GCVS, Kholopov et al. 1982), the Set of Identifications, Measurements and Bibliography for Astronomical Data (SIMBAD-version June 1993), the Database of UV Cet Type Flare Stars and Related Objects (Gershberg et al. 1993). The information on stellar magnitude at minimum and maximum given in the GCVS is not sufficient for assigning flare event parameters. Very often, the lack of identification charts is the reason for some misidentifications and errors (Tsvetkova \& Tsvetkov 1989). Approximately $20 \%$ of the UV Cet type stars included in the FSDB are not present in SIMBAD. For other flare stars, stellar magnitudes and coordinates are not given in SIMBAD. Very often only one reference is given, usually the Name-List of Variable Stars is quoted. SIMBAD is more complete for UV Cet type stars in 
the solar neighbourhood. We find the given pointer to SIMBAD in these cases very useful. Gershberg et al.'s (1993) database contains the data of 230 UV Ceti type stars mainly from the solar neighbourhood and some related objects.

\section{Future Developments}

The collection of data for all observed flare events in the solar neighbourhood and the application of various statistical methods to the data in the FSDB are the next steps. A serious obstacle for the FSDB is the lack of some necessary parameters of the flare event (beginning of event, duration etc.) in the literature of some stars. A further step will be the display of flare star identification charts, based on scans with a HP ScanJet IIcx. This is especially important for astrometric work, which now reaches much fainter magnitudes, but usually does not yet include flare stars (exceptions are the Pleiades flare stars and the work of Stauffer et al. 1991 and Prosser et al. 1991).

Acknowledgements: This project was supported by the Bulgarian National Science Foundation (projects F-340/93 and F-311/93), the Alexander von Humboldt Foundation, and the Deutsche Forschungsgemeinschaft. This research made use of the SIMBAD database, operated at CDS, Strasbourg, France.

\section{References}

Brand J., Wouterloot J.G.A., 1991, in Low Mass Star Formation in Southern Molecular Clouds, ESO Scientific Report No. 11, B. Reipurth (ed.), p. 1

Gershberg R.E., Shakhovskaya N.I., Katsova M.M., 1993,IBVS 3939

Haro G., Chavira E., Gonzales G., 1982, Bol. Inst. Tonantzintla, 3, No. 1, 3

Kholopov P.N. (ed.), 1985, General Catalogue of Variable Stars, Nauka, Moscow

Natsvlishvili R.Sh., 1991, Astrofiz. 31, 107

Prosser C.F., Stauffer J., Kraft R., 1991, AJ 101, 1361

Stauffer J., Klemola A., Prosser C.F., 1991, AJ 101, 980

Tsvetkov M., Chukova M., Tsvetkova K., 1994, in Proc. IAU Symp. 161, Astronomy from Wide-field Imaging, H.T. MacGillivray et al. (eds.), Kluwer, Dordrecht, p. 380

Tsvetkova K.P., Tsvetkov M.K., 1989, IBVS 3366 